leading to the formation of these autoantibodies. At present it is unkown how AMPA are generated and how autoreactive B-cell responses against PTM proteins are induced.

Objectives: To investigate how PTM proteins, more specifically carbamylated proteins, could contribute to a breach of B-cell tolerance.

Methods: Serum reactivity towards five different carbamylated proteins was determined for 160 RA-patients and 40 healthy individuals. Anti-CarP antibody cross-reactivity was studied by inhibition experiments. Mass spectrometry was performed to identify carbamylated self-proteins in human rheumatic and osteoarthritic joint tissue. Mice were immunized with carbamylated- or non-modified foreign (OVA) and self-antigens (mouse Albumin). Sera of immunized mice and monoclonal antibodies were analyzed for antigen reactivity.

Results: We show that anti-CarP antibodies in RA are highly cross-reactive towards multiple carbamylated proteins, including modified self- as well as modified non-self proteins. Immunization with carbamylated foreign proteins (Ca-OVA) induced a strong anti-CarP response against both carbamylated foreign- and self-proteins. Similar to murine serum anti-CarP antibodies, murine monoclonal anti-CarP antibodies were highly specific and cross-reactive to multiple carbamylated (auto)antigens. Although citrulline greatly resembles homocitrulline residues in structure, murine anti-CarP antibodies differ in antigen recognition profile from ACPA as they are able to discriminate between citrullinated and homocitrullinated forms of the same protein. Interestingly, we were able to identify carbamylated-albumin, in RA synovial tissue, indicating that albumin is present in carbamylated form locally in the inflamed joint.

Conclusions: Self-reactive AMPA-responses can be induced by exposure to foreign proteins containing PTM. Our findings show that autoreactive B-cell responses against PTM self proteins can be induced by exposure to PTM foreign proteins and provide new insights on the breach of autoreactive B-cell tolerance by foreign proteins.

Disclosure of Interest: None declared

DOI: 10.1136/annrheumdis-2017-eular.5984

\section{SAT0031 DETECTION AND ISOLATION OF ANTIGEN SPECIFIC B CELLS IN PATIENTS WITH RHEUMATOID ARTHRITIS}

J. Nilsson ${ }^{1}$, M. Bergquist ${ }^{1}$, K. Önnheim ${ }^{1}$, I.-L. Mårtensson ${ }^{1}$, J. Viljanen ${ }^{2}$, E. Lönnblom ${ }^{3}$, B. Xu ${ }^{3}$, O. Sareila ${ }^{3}$, J. Kihlberg ${ }^{2}$, R. Holmdahl ${ }^{3}$, I. Gjertsson ${ }^{1}$. ${ }^{1}$ Rheumatology and Inflammation Research, Gothenburg University, Göteborg; ${ }^{2}$ Department of Chemistry, BMC, Uppsala University, Uppsala; ${ }^{3}$ Medical Inflammation Research, Dept of medical Biochemistry and biophysics, Karolinska Institute, Stockholm, Sweden

Background: The majority of autoimmune diseases, e.g. rheumatoid arthritis (RA), are characterized by autoantibodies that are produced by B cells. We have identified several novel post-translationally modified epitopes in collagen type II (CII), a major cartilage constituent that are autoreactive. In mouse models, autoantibodies that bind to these epitopes can either induce or protect against inflammatory arthritis. However, very little is known about the frequencies or phenotype the $B$ cells that produce these autoantibodies in humans.

Objectives: The aim of this project is to study the B cells that produce autoantibodies reactive to Cll-epitopes in patients with RA.

Methods: Clinical data and peripheral blood were collected from patients with RA $(n=100)$. Titres of Cll-reactive serum-autoantibodies were determined by Luminex. The different subsets of $B$ cells that expressed a Cll-reactive B cell receptor (BCR) were analysed and isolated in patients with positive $(n=10)$ or negative $(n=5)$ titres for autoantibodies recognizing the Cll-epitopes cyc48 (CII-F4-R-Cit) and cyc49 (CII-F4-Cit-R) as well as in healthy controls $(n=3)$ using flow cytometry. Results: In patients with detectable autoantibodies to cyc48 and cyc49, their titres were $13761 \pm 3815$ and $5585 \pm 1992$ (mean \pm SEM), respectively. The corresponding titres in the patients negative for these autoantibodies were $122 \pm 55$ and $234 \pm 75$. In autoantibody-positive patients, the frequencies of cells expressing a BCR reactive for cyc48 and cyc49 in different subsets were: transitional cells $0.88 \pm 0.18 \%$ and $1.33 \pm 0.33 \%$; naïve cells $0.09 \pm 0.0008 \%$ and $0.13 \pm 0.06$ and memory cells $0.31 \pm 0.25$ and $0.30 \pm 0.12 \%$. All frequencies were well above those detected in patients without cyc48/49 titres and in healthy controls. A higher proportion $(p=0.03)$ of the cyc48/49 positive patients were treated with methotrexate compared to cyc48/49 negative patients, no other clinical differences were recorded. Cll-reactive single $B$ cells were isolated in order to clone the BCR

Conclusions: In patients with manifest RA there is a relatively high and detectable frequency of transitional $B$ cells that express a joint-specific BCR, which suggests that the deletion of autoreactive $B$ cells in the bone marrow in RA patients is defective. As there is a decrease in the proportion of naïve Cll-specific B cells, a substantial amount of the autoreactive cells are deleted in the periphery. However, peripheral B-cell tolerance is incomplete, as Cll-specific B cells are enriched in the pool of memory B cells.

Disclosure of Interest: None declared

DOI: 10.1136/annrheumdis-2017-eular.3680

\section{SAT0032 EX VIVO ANALYSIS OF AUTOANTIGEN-SPECIFIC T CELL RESPONSES USING A MULTI HLA-CLASS II TETRAMER APPROACH}

C. Gerstner ${ }^{1}$, H. Uchtenhagen ${ }^{1,2}$, A. Dubnovitsky ${ }^{3,4}$, W. Kwok ${ }^{2}$, E.A. James ${ }^{5}$, V. Malmström ${ }^{1} .{ }^{1}$ Rheumatology Unit, Department of Medicine Solna, Karolinska Institutet, Stockholm, Sweden; ${ }^{2}$ Translational Research program, BRI at Virginia Mason, Seattle, United States; ${ }^{3}$ Department of Clinical Neurosciences; ${ }^{4}$ Science for Life Laboratory, Department of Medicine Solna, Karolinska Institutet, Stockholm, Sweden; ${ }^{5}$ Tetramer Core, BRI at Virginia Mason, Seattle, United States

Background: HLA class II tetramers allow the direct ex vivo enumeration and phenotypic characterization of antigen-specific T cells and have proven a useful tool in different settings, e.g. allergy and vaccination.

In rheumatoid arthritis (RA), many candidate auto-antigens have been characterized and are believed to play a role in the disease.

Objectives: Based on flow cytometry we developed a multi HLA-class II tetramer approach that renders it possible to look at numerous specificities simultaneously and is sufficiently sensitive at the same time as auto-reactive $T$ cells are likely to be rare. We focused on validated citrullinated $T$ cell epitopes previously studied in RA, namely a-enolase, fibrinogen and CILP.

To test the robustness and sensitivity of our multi-tetramer assay, we performed repeated experiments on PBMCs of HLA-DRB1*04:01-positive healthy controls as well as HLA-DRB1*04:01-positive RA patients.

Results: First, we examined the sensitivity of the panel by assessing PBMC from healthy donors and we could detect low frequencies of auto-reactive T cells in these samples (1-10 per million CD4), mostly displaying a naïve phenotype, which was in sharp contrast to influenza-specific T cells in the same donors which were 10-20 fold higher in numbers and of memory phenotype. Next, we tested the robustness of the panel by running technical repeats of all healthy donor samples, which yielded similar frequencies. Thereafter, we focused on RA patient PBMC obtained from repeated blood draws (2-4 weeks apart). Also in these samples, frequencies ranged between 1-10 tetramer-positive cells per million CD4+ T cells, i.e. similar as in healthy controls. Moreover, not all T cell specificities were present in all patients. Still, we found the frequencies to be stable in the repeated blood draws in approximately half of the individuals, implicating that frequencies close to 1 cell per million CD4+ T cells is borderline of what we can stably detect. Importantly, the patient samples utilized were not taken from time points of active disease, where we hypothesize frequencies to be elevated. This may be the case also in synovial fluid and as a proof of principle, we assessed synovial fluid samples of HLA-DRB1 ${ }^{*} 04: 01+$ RA patients and were able to detect auto-reactive $T$ cells at frequencies comparable to the ones found in peripheral blood.

Conclusions: In conclusion, we developed a sensitive tetramer panel allowing the simultaneous enumeration and phenotypic characterization of antigen-specific $T$ cells in ex vivo samples from RA patients that can be used for monitoring and in-depth studying of these cells during the course of disease and treatment in individual patients.

Disclosure of Interest: None declared

DOI: 10.1136/annrheumdis-2017-eular.2796

\section{SAT0033 SMOKING CONTRIBUTES TO EXHAUSTED STATE OF CD4+ T CELLS IN RHEUMATOID ARTHRITIS}

C. Wasén, M. Turkkila, M. Erlandsson, K.M. Andersson, M. Brisslert, S. Töyrä Silfverswärd, M.I. Bokarewa. Department of Rheumatology and Inflammation Research, University of Gothenburg, Gothenburg, Sweden

Background: Rheumatoid arthritis (RA) has recently been linked to an exhausted state of $\mathrm{CD}^{+} \mathrm{T}$ cells in peripheral blood of patients [1]. Exhaustion of CD4 ${ }^{+} \mathrm{T}$ cells limits their proliferation and increases cell death. In $\mathrm{CD} 8^{+} \mathrm{T}$ cells smoking counteract exhaustion, which may lead to increased cytotoxic activity exemplified by targeting of cells with high expression of the anti-apoptotic protein survivin [2]. Exhaustion of CD4 ${ }^{+} \mathrm{T}$ cells coincides with expression of interferon (IFN) response genes [3], referred to as the IFN signature. The development of the IFN signature has been suggested to predate RA [4].

Objectives: We investigated how smoking affect the $\mathrm{CD}^{+} \mathrm{T}$ cell population in peripheral blood of RA patients with focus on the exhaustion marker programmed cell death-1 (PD-1).

Methods: Blood samples were collected from RA patients and healthy women with different smoking status and analysed for PD-1 and survivin expression using flow cytometry and qPCR. Sorted Th17 cells from peripheral blood were analysed for expression of 18 genes up regulated during exhaustion [3], herein referred to as the exhaustion set, and serum levels of survivin were assessed by ELISA. Peripheral blood CD4+ cells were analysed for their expression of seven IFN response genes [4]. The role of survivin in the formation of exhausted CD4 ${ }^{+}$ $T$ cells was studied in collagen-induced arthritis $(\mathrm{CIA})$, where mice were treated with nicotine or vaccinated with survivin peptides.

Results: High frequency of exhausted PD $-1^{+} \mathrm{CD} 4^{+}$cells was found in smoking RA patients. The numbers of $\mathrm{PD} 1^{+} \mathrm{CD} 4^{+}$cells correlated inversely with the PD-1 expression by cytotoxic $C D 8^{+} C D 107^{+}$cells $(r=-0.62, p=0.01)$. Additionally, the frequency of $\mathrm{PD}-1^{+} \mathrm{CD} 4^{+}$cells increased with reduction of the $\mathrm{CD} 4^{+}$population $(r=-0.71, p=0.002)$. The IFN signature was found exclusively among smoking RA patients. The patients with the IFN signature all had $C D 4^{+}$cells with low survivin 